\title{
On the Role of External and Internal Irreversibilities towards Classical Entropy Generation Predictions in Equilibrium Thermodynamics and their Relationship with Heat Transfer
}

\author{
Suman Chakraborty*
}

Department of Mechanical Engineering, Indian Institute of Technology, Kharagpur-721302, India

\begin{abstract}
In classical equilibrium thermodynamics description, ambiguities exist in terms of pinpointing external and internal irreversibilities in overall entropy generation prediction, as a system undergoes a thermodynamic process. The present work attempts to bridge this gap between classical thermodynamics-based irreversibility predictions and finite time heat transfer analysis. By choosing a model problem, expressions for external irreversibilities are quantitatively derived and it is observed that ambiguities may exist in the pertinent quantification depending on the very definition of system, immediate surroundings and surroundings. It is observed that if variations within immediate surroundings are taken into account, more realistic estimates of external irreversibilities can be obtained in equilibrium thermodynamics framework, as a function of heat transfer characteristics of the same. It is also shown that for some special cases, different expressions for external irreversibilities asymptotically converge to the same entropy generation predictions, in effect.
\end{abstract}

\section{INTRODUCTION}

As a consequence of the second law of thermodynamics, the sum total of change in entropy of the system and surroundings (i.e., for the Universe, in totality) is often expressed in equilibrium thermodynamics (classical) formulation as [1-3]:

$$
S_{2}-S_{1}=\int_{1}^{2} \frac{\delta Q}{T}+S_{g e n}
$$

where $\delta \mathrm{Q}$ represents heat transfer across the system boundary (at local absolute temperature $\mathrm{T}$ ) and $\mathrm{S}$ represents the entropy. In the above equation, subscripts 1 and 2 represent the end thermodynamic states for the process under consideration, and subscript 'gen' represents a generation (source) term that is supposed to be always greater than or equal to zero (principle of increase of entropy). Although in standard thermodynamics texts the above-mentioned entropy generation is generally mentioned as a consequence of irreversibilities [1-3], specific origin and direct quantitative measure of the same is nowhere pinpointed, leading to several ambiguities. For example, in many situations, it is not explicitly mentioned whether it is due to the total irreversibilities (internal + external) or internal irreversibilities alone. Even though scientific intuition clearly suggests that it is supposed to be a measure of internal irreversibilities within the system, the very notion of 'system' here may turn out to be quite confusing. Resolution of such anomalies is by no means an obvious task, since the fact whether external irreversibilities are absorbed within the term $\int_{1}^{2} \frac{\delta Q}{T}$ or not solely depends on

*Address correspondence to this author at the Department of Mechanical Engineering, Indian Institute of Technology, Kharagpur-721302, India; E-mail: suman@mech.iitkgp.ernet.in the exact definition of the pertinent thermodynamic systems and their respective surroundings. In that context, ambiguities often arise in the notion of the terms 'surroundings' and 'immediate surroundings'. Subtle differences between the various entropy generation predictions, therefore, may exist in accordance with the thermodynamic entities included in the 'system', 'immediate surroundings' and 'surroundings' themselves. Aim of the present work is to develop an unified approach in equilibrium thermodynamics framework to resolve the associated fallacies by outlining convenient means of pinpointing entropy generation due to internal and external irreversibilities, thereby, bridging some of the missing links between equilibrium thermodynamics and heat transfer.

\section{ANALYSIS}

In order to make a critical assessment of the parameters mentioned as above, let us refer to a model thermodynamic system (say, a substance enclosed in a piston-cylinder arrangement for example, refer to Fig. 1) interacting with its surroundings by means of total heat transfer $Q$ across an area A. The heat is effectively transferred from the ambient (or equivalently, some other heat source) having a constant temperature of $\mathrm{T}_{\infty}$. In order to reach the substance, thermal energy supplied by the source basically has to overcome two specific thermal resistances, namely, convective resistance between the ambient and the cylinder wall and conductive resistance within the wall of the cylinder itself (wall thickness $=\delta$ ). Consequently, the instantaneous temperature at ambient-wall interface (i.e., $\mathrm{T}_{0}$ ) and wall-substance interface (i.e., T) are different from the 'surroundings' temperature $\mathrm{T}_{\infty}$. However, the substance comprising the thermodynamic system under concern can assumed to be at a state of thermodynamic equilibrium (i.e., change in state of the system is modelled by a so-called 'quasiequilibrium' or 'quasi-steady' process) with a consequence that at the thermodynamic state under consideration the entire system is in thermal equilib- 
rium as well. On the other hand, temperature differences are likely to exist across the two end faces of the cylinder-wall for the heat to be conducted across the same, and therefore, the wall is never in thermodynamic equilibrium. In fact, the very notion of thermodynamic equilibrium in the wall would preclude any possibility of heat transfer across it (since, heat is energy in transient by virtue of temperature difference). Therefore, if the wall is considered to be a part (or, an extension) of the system under investigation, it cannot be analyzed in the light of equilibrium thermodynamics, since thermal equilibrium necessarily demands a uniformity of temperature throughout the system at any thermodynamic state during the process. This might, however, give rise to ambiguous entropy generation predictions. In order to resolve the situation, we attempt here to make a combined 'equilibrium thermodynamics' and 'heat transfer' analysis of the situation, thereby pinpointing the nature of irreversibilities predicted through the statement of equation (1). In order to simplify the problem mathematically without losing pertinent physical features, we make the following assumptions for the present analysis:

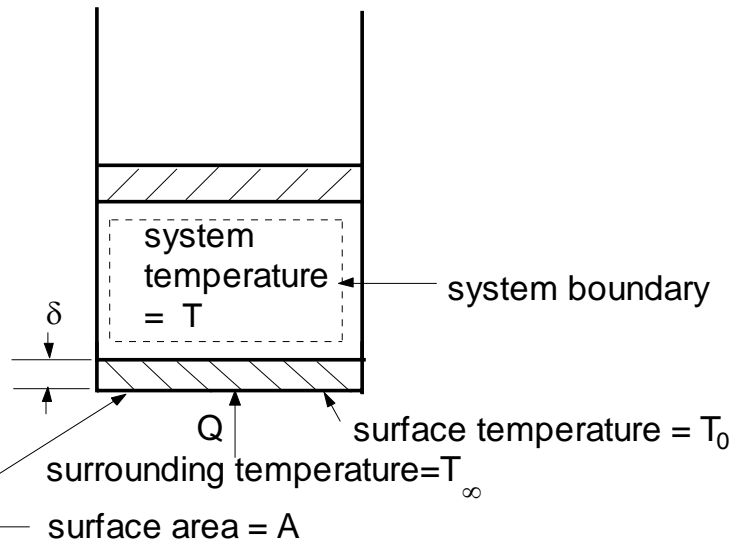

Fig. (1). A schematic diagram of the model situation considered for analysis.

(i) The heat flux across the system boundary is timeinvariant.

(ii) The behaviour of the system itself is transient, which, for simplicity, is considered to be a lumped mass of equivalent specific heat capacity $\mathrm{C}$ and mass $\mathrm{m}$.

Under the above assumptions, from energy conservation principles applied for the physical problem depicted in Fig. (1), we can write:

$\dot{Q}=A h\left(T_{\infty}-T_{0}\right)=\frac{k A\left(T_{0}-T\right)}{\delta}=m C \frac{d T}{d t}$

where $\mathrm{h}$ is the convective heat transfer coefficient between the cylinder wall outer surface and the ambient. Although radiation heat transfer is not explicitly considered in the present analysis to avoid mathematical complications, it can be equivalently absorbed in the heat transfer rate equation by formulating an effective heat transfer coefficient $h_{\text {eff }}$ such that $h_{\text {eff }}=h+\sigma \varepsilon\left(T_{\infty}^{2}+T_{0}^{2}\right)\left(T_{\infty}+T_{0}\right), \quad \sigma$ being the
Stefan-Boltzmann constant, and $\varepsilon$ being the emissivity. Equation (2) can be conveniently rewritten as

$\frac{T_{0}-T}{\delta / k A}=\frac{T_{\infty}-T_{0}}{1 / A h}=\frac{T_{\infty}-T}{\delta / k A+1 / A h}=m C \frac{d T}{d t}$

The above can be written in a compact form of:

$m C \frac{d \theta}{d t}+\frac{\theta}{R}=0$

where $\theta=T-T_{\infty}, \quad R=\delta / k A+1 / A h \quad$ (overall resistance). Integrating equation (4) from an initial thermodynamic state $\left(\mathrm{t}=0, \mathrm{~T}=\mathrm{T}_{1}\right)$ to a final state $(\mathrm{t}=\mathrm{t}, \mathrm{T}=\mathrm{T})$ we get

$T-T_{\infty}=\left(T_{1}-T_{\infty}\right) \exp (-t / m C R)$

Thus,

$\int \frac{\delta Q}{T}=m C \ln \left|\frac{T_{\infty}+\left(T_{1}-T_{\infty}\right) \exp (-t / m C R}{T_{1}}\right|$

At this stage, one needs to appreciate that a major source of ambiguity here is whether to take the term ' $\mathrm{T}$ ' in the expression $\int \frac{\delta Q}{T}$ as temperature of the actual system boundary (i.e., $\mathrm{T}$ ) or the extended system boundary (i.e., $\mathrm{T}_{0}$ ), or the thermal reservoir temperature (i.e., $\mathrm{T}_{\infty}$ ). To assess implications of each of the corresponding estimations as per above considerations, we subsequently calculate $\int \frac{\delta Q}{T_{0}}$ and $\int \frac{\delta Q}{T_{\infty}}$ as follows:

$\int \frac{\delta Q}{T_{0}}=m C(1+B i) \ln \left|\frac{T_{\infty} B i+T_{\infty}+\left(T_{1}-T_{\infty}\right) \exp (-t / m C R}{T_{\infty} B i+T_{1}}\right|$

where $B i=h \delta / k$.

$\int \frac{\delta Q}{T_{\infty}}=m C \frac{T-T_{1}}{T_{\infty}}=$

$\frac{m C\left(T_{\infty}-T_{1}\right)[1+\exp (-t / m C R)]}{T_{\infty}}$

Now, since for positive $\delta Q, \mathrm{~T}_{\infty}>\mathrm{T}_{0}>\mathrm{T}$, we can write:

$\int_{1}^{2} \frac{\delta Q}{T}>\int_{1}^{2} \frac{\delta Q}{T_{0}}>\int_{1}^{2} \frac{\delta Q}{T_{\infty}}$

The inequalities expressed by expression (9) can be written as 'equivalent' equalities as: 
$\int_{1}^{2} \frac{\delta Q}{T}=\int_{1}^{2} \frac{\delta Q}{T_{0}}+\left.S_{g e n}\right|_{T \rightarrow T_{0}}$

$\int_{1}^{2} \frac{\delta Q}{T}=\int_{l}^{2} \frac{\delta Q}{T_{\infty}}+\left.S_{g e n}\right|_{T \rightarrow T_{\infty}}$

$\int_{1}^{2} \frac{\delta Q}{T_{0}}=\int_{1}^{2} \frac{\delta Q}{T_{\infty}}+\left.S_{g e n}\right|_{T_{0} \rightarrow T_{\infty}}$

In order to assess the 'total' irreversibility, we can also write:

$$
\begin{aligned}
& \int_{1}^{2} d S_{\text {system }}=S_{2}-S_{1} \\
& \int_{1}^{2} d S_{\text {surroundings }}=-\int_{1}^{2} \frac{\delta Q}{T_{\infty}}
\end{aligned}
$$

as per standard thermodynamics notations. Implicit to the above considerations is the fact that the immediate surroundings (i.e., the container-wall in the present example) are taken to parts of the system itself, and thermal variations within immediate surroundings are essentially neglected (which is a logical assumption provided thermal mass of the wall is negligible in comparison to that of the actual system). Although authenticity of this is by no means beyond question, there is very little that one can do about it, since temperature variations within a sub-part of the system cannot be accommodated within framework of equilibrium thermodynamic considerations. Now, adding equations (13) and (14) we can obtain:

$$
\begin{aligned}
& \Delta S_{\text {universe }}=\Delta S_{\text {system }}+\Delta S_{\text {surroundings }} \\
& =S_{2}-S_{1}-\int_{1}^{2} \frac{\delta Q}{T_{\infty}} \\
& \text { i.e., } S_{2}-S_{1}=\int_{1}^{2} \frac{\delta Q}{T_{\infty}}+\Delta S_{\text {universe }}
\end{aligned}
$$

Thus, comparing equations (1) and (15) we can see that $S_{\text {gen }}=\Delta S_{\text {universe }}$ if $T$ is taken to be $T_{\infty}$ in equation (1). However, underlying implication of $\mathrm{T}=\mathrm{T}_{\infty}$ would be the absence of any external irreversibilities in the sense that total heat transfer $\mathrm{Q}$, in that case, occurs across an infinitesimally small temperature difference $\Delta \mathrm{T}$ between surroundings at temperature $\mathrm{T}_{\infty}$ and system at a temperature $\mathrm{T}=\mathrm{T}_{\infty}-\mathrm{dT}$ (where $\mathrm{dT} \rightarrow 0$ ). In that case, the term $\mathrm{S}_{\text {gen }}$ would represent only the internal irreversibilities present in the system (due to absence of any external irreversibilities). In the same situation, however, if we use equation (1) retaining the original meaning of the term $\int_{1}^{2} \frac{\delta Q}{T}, \mathrm{~S}_{\mathrm{gen}}$ would include both the effects of external and internal irreversibilities. $S_{2}-S_{1}$ being a term dependent solely on the specified end states of the system, the above implies that the expression (refer to equation 11)

$$
\int_{1}^{2} \frac{\delta Q}{T}-\int_{1}^{2} \frac{\delta Q}{T_{\infty}}=\left.S_{g e n}\right|_{T \rightarrow T_{\infty}}
$$

is a measure of external irreversibilities associated with the process. Under the specific assumptions made for the illustrative example taken up in this study, the above can be quantified as (using equations 6-8)

$$
\begin{aligned}
& \left.S_{g e n}\right|_{T \rightarrow T_{\infty}}=m C \ln \left|\frac{T_{\infty}+\left(T_{1}-T_{\infty}\right) \exp (-t / m C R}{T_{1}}\right| \\
& -m C \frac{\left(T_{\infty}-T_{1}\right)[1-\exp (-t / m C R)]}{T_{\infty}}
\end{aligned}
$$

$$
\text { As } \mathrm{t} \rightarrow \infty \text {, equation (17) would yield }
$$

$$
\left.S_{g e n}\right|_{T \rightarrow T_{\infty}}=m C\left[\ln \left|\frac{T_{\infty}}{T_{1}}\right|+\frac{T_{1}}{T_{\infty}}-1\right] \geq 0
$$

(since, $T_{\infty}>T_{1}$ for the heat transfer $Q$ to take place in the sense as assumed for the model test problem here). It can be noted here that equation (18) is based on the consideration of immediate surroundings not as a part of the system analysis. On the other hand, if immediate surroundings are included within the system itself (but heat capacity of the immediate surroundings are neglected) then the term $\mathrm{T}$ in equation (10) can be replaced by $\mathrm{T}_{0}$ (which is, in general, a variable), and then, the measure of external irreversibilities would be given by equation (12) (i.e., an expression for $\left.S_{g e n}\right|_{T_{0} \rightarrow T_{\infty}}$ ). For the model situation under consideration, this reduces to (using equations (7) and (8))

$$
\begin{aligned}
& \left.S_{g e n}\right|_{T_{0} \rightarrow T_{\infty}}= \\
& m C(1+B i)^{*} \\
& \ln \left|\frac{T_{\infty} B i+T_{\infty}+\left(T_{1}-T_{\infty}\right) \exp (-t / m C R}{T_{\infty} B i+T_{1}}\right|- \\
& m C \frac{T_{\infty}-T_{1}}{T_{\infty}}[1-\exp (-t / m C R)]
\end{aligned}
$$

As $t \rightarrow \infty$, equation (19) would yield

$$
\begin{aligned}
& \left.S_{g e n}\right|_{T_{0} \rightarrow T_{\infty}}=m C(1+B i) \ln \left|\frac{T_{\infty} B i+T_{\infty}}{T_{\infty} B i+T_{1}}\right| \\
& -m C \frac{T_{\infty}-T_{1}}{T_{\infty}} \geq 0
\end{aligned}
$$

Clearly, expression (20) appears to be a more appropriate measure of external irreversibilities, since it includes effects 

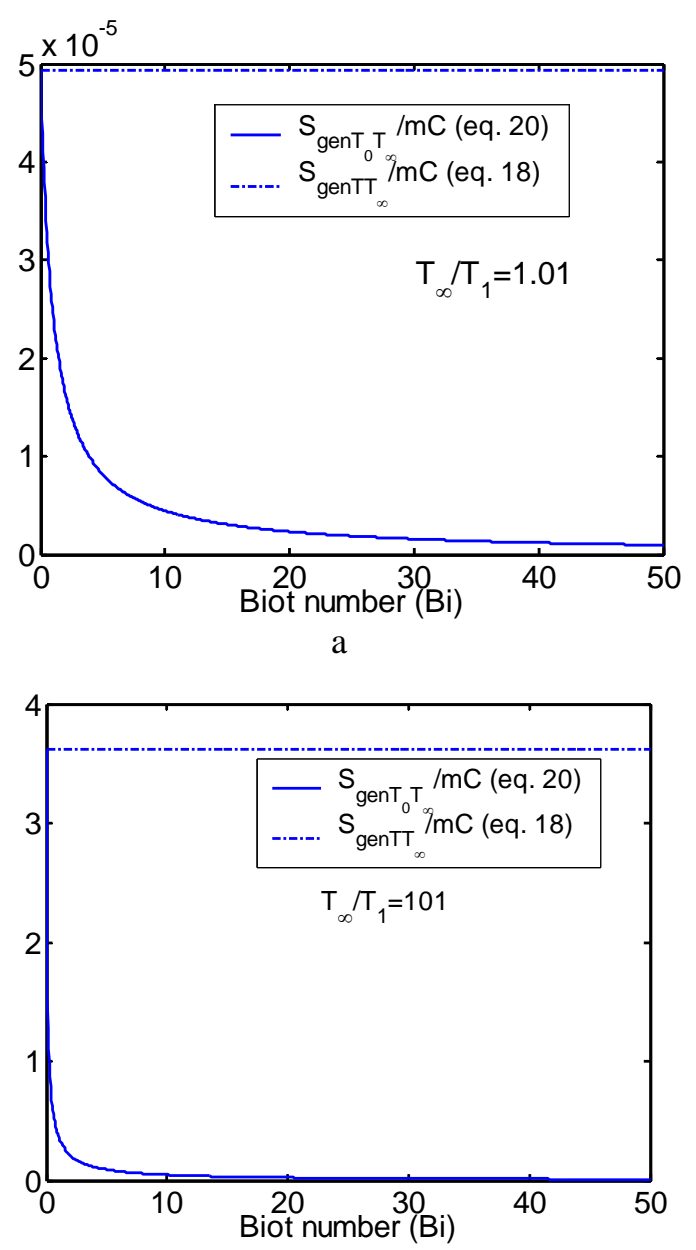

$\mathrm{b}$

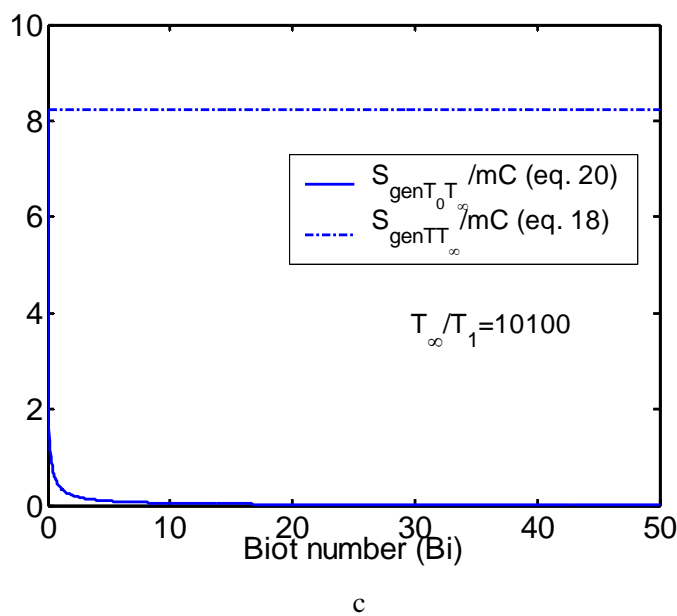

Fig. (2). Entropy generations predicted by equations (18) and (20): (a) $\mathrm{T}_{\alpha} / \mathrm{T}_{1}=1.01$ (b) $\mathrm{T}_{\alpha} / \mathrm{T}_{1}=101$ (c) $\mathrm{T}_{\alpha} / \mathrm{T}_{1}=10100$.

of the immediate surroundings into account by incorporating the Biot number corresponding to the cylinder thickness across which the heat is being conducted to the system. Expressions for external irreversibility, as depicted by equations (20) and (18), are plotted in Fig. (2). It is clearly observed that for a specified value of $\mathrm{T}_{\infty} / \mathrm{T}_{1}$, as Bi decreases, external irreversibility depicted by equation (20) progressively increases, and the two entropy generation predictions asymptotically approach each other as $\mathrm{Bi} \rightarrow 0$. This can be attributed to the fact that a lower Biot number essentially implies a smaller ' $h$ ' for a fixed ' $\delta$ ' and ' $k$ '. A smaller ' $h$ ', in turn, implies a larger temperature difference to achieve the same rate of overall heat transfer across the system boundary. A larger temperature difference effectively results in greater external irreversibilities as predicted by the corresponding entropy generation. It can also be inferred here as a corollary that although both expressions (18) and (20) are measures of external irreversibilities in some sense, they are not identical until and unless, either:

(i) $\quad \mathrm{T}_{\infty}=\mathrm{T}_{1}$ (irrespective of the value of $\mathrm{Bi}$ ), which would then mean

$\left.S_{g e n}\right|_{T \rightarrow T_{\infty}}=\left.S_{g e n}\right|_{T_{0} \rightarrow T_{\infty}}=0$

or, otherwise

(ii) $\quad \mathrm{Bi} \rightarrow 0$

Equation (21) essentially represents the trivial case of an externally reversible process actuated by an infinitesimally small temperature difference between the thermal reservoir and the system at its initial state. Since all subsequent thermodynamic states of the system should also comply with the same condition for overall external reversibility, it follows that an infinitesimally small temperature difference subsequently needs to be maintained between system temperature (T) and surroundings temperature $\left(\mathrm{T}_{\infty}\right)$ throughout the process. The reservoir temperature $\left(\mathrm{T}_{\infty}\right)$ being fixed in this example, this will imply that the process needs to be isothermal in nature (for the special case), ensuring satisfaction of 'internal reversibility' conditions as well, on account of inherent 'slowness' of such processes for internal readjustments required to achieve thermodynamic equilibrium in each intermediate state through which the system passes. This establishes a kind of interlinkage between the conditions of external reversibility and internal reversibility in a model thermodynamic process. However, such 'idealized' processes are far from practical in nature, and in reality, both types of irreversibilities are very much present. While the expression $\left(S_{2}-S_{1}\right)-\int_{1}^{2} \frac{\delta Q}{T_{\infty}}$ represents internal irreversibilities for the thermodynamic process, the external irreversibilities can be estimated by expressions such as equations (18) or (20), depending upon the specific pinpointed definition of system, immediate surroundings and surroundings adopted for thermodynamic analysis. It can be noted that for $\mathrm{Bi} \rightarrow 0$ (equation 22 ), the conductive resistance is virtually absent across the cylinder wall in comparison to the resistance against 'external' heat transfer, and consequently, entropy generation predictions including and excluding immediate surroundings in the overall system analysis effectively become identical in that case.

\section{CONCLUSIONS}

Particular care needs to be taken to couple finite time heat transfer considerations with equilibrium thermodynam- 
ics based external irreversibility predictions; since the very notion of thermodynamic equilibrium of a system would preclude any chance of heat transfer. The present work attempts to bridge this gap and proposes guidelines for model thermodynamic calculations quantifying external irreversibilities associated with the process, given a specific definition of the thermodynamic system used for the analysis. It is shown that different external irreversibility predictions asymptotically converge to the same as the Biot number characterizing heat transfer behaviour of the immediate surround- ings approaches zero for the specific case under investigation.

\section{REFERENCES}

[1] R. E. Sonntag, C. Borgnakke, and G. J. Van Wylen, Fundamentals of Thermodynamics. Singapore: John Wiley and Sons, 2002.

[2] M. J. Moran, and H. N.Shapiro, Fundamentals of Engineering Thermodynamics, Singapore: John Wiley and Sons, 2003.

[3] Y. A. Cengel, and M. Boles, Thermodynamics, An Engineering Approach, New York: McGraw-Hill, 2001. permits unrestrictive use, distribution, and reproduction in any medium, provided the original work is properly cited. 